\title{
Surgical fixation of rib fractures: how I do it
}

\author{
Gregor J. Kocher ${ }^{1}$, Mohammad Al-Hurani ${ }^{1}$, Fabrizio Minervini ${ }^{2}$ \\ ${ }^{1}$ Division of General Thoracic Surgery, Inselspital, Bern University Hospital, University of Bern, Bern, Switzerland; ${ }^{2}$ Department of Thoracic \\ Surgery, Kantonsspital Luzern, Lucerne, Switzerland \\ Contributions: (I) Conception and design: GJ Kocher, M Al-Hurani; (II) Administrative support: GJ Kocher, M Al-Hurani; (III) Provision of study \\ materials or patients: GJ Kocher, M Al-Hurani; (IV) Collection and assembly of data: All authors; (V) Data analysis and interpretation: All authors; (VI) \\ Manuscript writing: All authors; (VII) Final approval of manuscript: All authors. \\ Correspondence to: Gregor J. Kocher. Division of General Thoracic Surgery, University Hospital Bern, CH - 3010 Bern, Switzerland. \\ Email: gkocher@gmx.ch.
}

\begin{abstract}
Chest wall trauma is common worldwide. The majority of cases are a result of blunt mechanisms. Among these injuries, rib fractures are considered the most frequent type of clinically significant blunt thoracic trauma sequelae and are associated with considerable morbidity and mortality. Despite a large number of cases annually, there are no internationally recognized guidelines for rib fracture treatment yet. This controversy in treatment options can be attributed to the lack of high-level convincing clinical research that confirms the benefits of surgical treatment over conservative treatment measures. Over the last 100 years, rib fixation has been carried out infrequently using non-specific materials due to lack of specific rib fixation systems. However, a substantial advancement in materials (e.g., low profile titanium plates) and technologies (e.g., angle-stable locking screws) was achieved in the last decade. Despite these developments, so far no clinical study has proven the superiority of one system over another. In this article, we would like to share our experience in this field including indications for surgical rib fixation and how we perform such procedures.
\end{abstract}

Keywords: Surgical rib fixation; flail chest; pseudarthrosis; video-assisted thoracic surgery (VATS); uniportal

Received: 29 August 2019; Accepted: 28 October 2019; Published: 05 April 2020.

doi: 10.21037 /jovs.2019.11.04

View this article at: http://dx.doi.org/10.21037/jovs.2019.11.04

\section{Introduction}

Chest wall trauma is very common worldwide and is seen in $28 \%$ of all trauma cases $(1,2)$. Subsequently rib fractures are of the most common traumatic injuries after chest wall trauma.

They result in significant pathophysiological changes that may lead to considerable morbidity and mortality (1). $\mathrm{Up}$ to $60 \%$ of rib fracture patients treated conservatively experienced morbidity by means of nosocomial pneumonia and prolonged ventilatory support, resulting in a longer hospital stay (3). Even after hospital discharge, $40-60 \%$ of patients cannot resume full-time employment because of chest pain (3), especially if complicated with non-union or intercostal nerve entrapment (4). Traditional conservative management aims at (I) adequate pain control (up to epidural analgesia) and (II) respiratory support (i.e., daily physiotherapy sessions, sometimes up to intubation and mechanical ventilation) (5-7).

Surgical rib stabilization has become increasingly important over the last decade (7). Besides restoring the morphology of the fractured ribs and with that the natural shape and function of the whole chest wall, surgical rib fixation aims at eliminating pathophysiological damage (6). Already many years ago, surgeons have started to carry out rib fixation, but these procedures were done rather infrequently, and had to be performed using nonspecific materials such as wires, sutures or common reconstruction plates (8). Recently, new material and technologies have been developed and enriched this field and more recent studies support surgical stabilization over conservative treatment, showing that the surgical option 

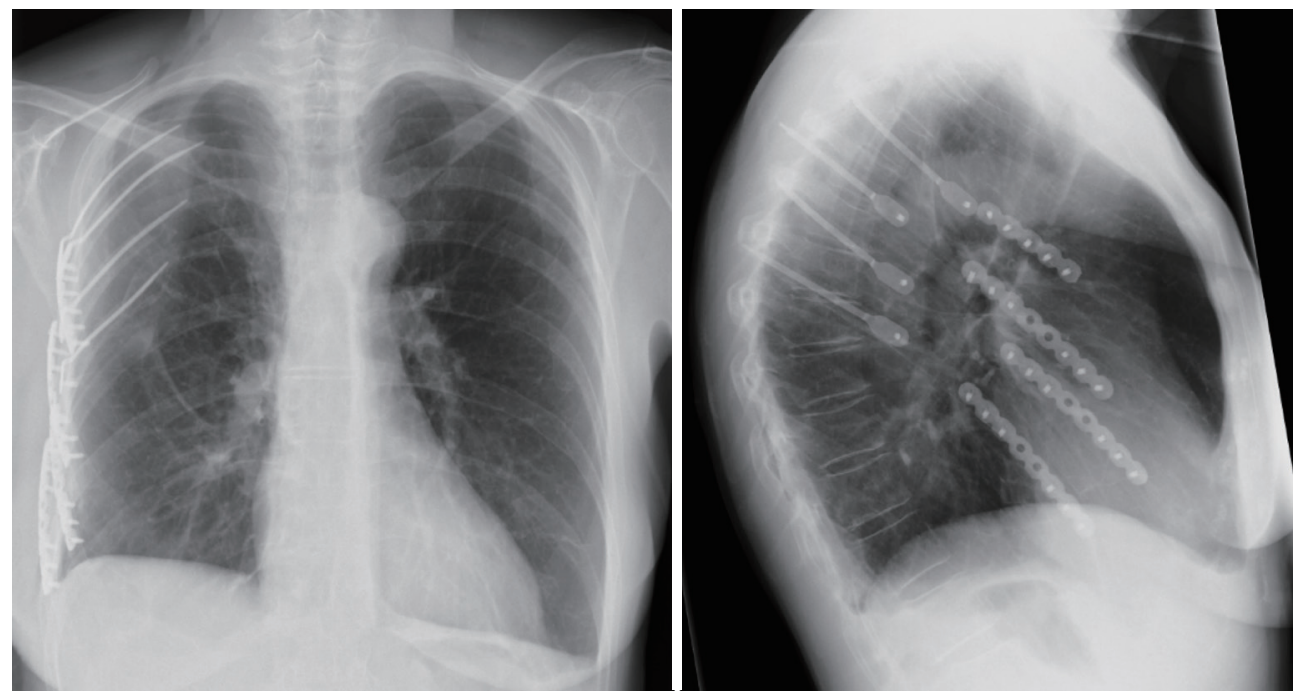

Figure 1 Flail chest stabilization with plate fixation of the anterior fracture row with titanium plates and additional fixation of the posterior fracture row with intramedullary splints $\left(\right.$ MatrixRib $\left.^{\mathrm{TM}}\right)$.

has a beneficial influence in terms of reduction of pain, duration of mechanical ventilation, hospital stay, ICU time, tracheostomy rate, and pulmonary infection rate (5,6,9-11).

Despite the growing interest in rib fracture fixation, there is still no internationally recognized consensus regarding indications and patient selection (7). Also, no method and/ or technique was found to be superior compared to others so $\operatorname{far}(12)$.

\section{Patient selection and workup}

Most rib fractures are not life-threatening and as such they can usually be treated non-operatively when fewer than three ribs are fractured, if there is no displacement, and if there are no associated organ injuries present $(1,4)$. Rib fixation seems to be most worthwhile in ventilator-dependent flail chest patients, if neurologically adequate (13). In addition, patients with three or more rib fractures, any feature of displacement, more than $15 \mathrm{~mm}$ overlap between fractured rib segments, or failed to taper from continuous intravenous analgesia, should be considered for surgical treatment $(1,3)$.

In surgically treated patients, fixation often must not be carried out in all fracture sites, rather one must seek a balance between surgical damage and fixation benefits (6). Moreover, fixation of ribs with obvious physiological hazards should be prioritized.

Because of the deep position of the ribs 1 to 3 , difficulty in exposure, limited contribution to the respiratory mobility, and because of some vital structures (i.e., neurovascular bundle) in close proximity, the surgical procedure carries a higher risk and is rarely indicated (6).

In addition, fractures of the free floating ribs are generally not recommended to be fixed due to difficult accessibility and minimal respiratory significance, unless associated with liver or spleen injuries $(1,6)$.

Chest wall stability is mainly upheld by the ribs 4 to 10 (6), which also contribute most significantly to the thoracic movement (9). So, any fracture of those ribs may lead to major friction between fractured segments and subsequently results in considerable pain (9). Nevertheless, it has to be noted that even though in a flail chest most ribs are broken in two places, it is often sufficient to stabilize only one of these two fractures per rib in order to restore an adequate stability of the chest wall. Only if the chest wall still shows significant instability after fixation of only one fracture site, or if we have to deal with multifragmented rib fractures, also the other fracture(s) of the same rib may need to be approached (see Figure 1).

As part of a secondary survey, all thoracic trauma patients should get a chest X-ray (12). However, this diagnostic modality can miss up to $50 \%$ of pneumothoraces and more than $50 \%$ of rib fractures $(12,14)$. Therefore, CT scan plays an essential role in diagnosing thoracic trauma injuries $(9,14)$. Moreover, it helps in planning surgery including the most appropriate site for the incision and the optimal 
position of the patient during surgical rib fixation (9).

\section{Pre-operative preparation}

After primary and secondary surveys, the patient's condition should be further optimized by efficient pain control and aggressive pulmonary hygiene (1). Furthermore, early prophylactic antibiotics administration-especially in case of open fractures - aids in infection prevention (10).

Recently published papers support early surgical stabilization (within 72 hours) $(1,4)$. Further, some recommend that the intervention should be ideally performed within 24 hours (1). The proven advantages of early rib fixation range from shorter ventilatory support, decreased risk of pneumonia, and shorter hospital stay when the patient is operated within 48 hours (15). Other benefits of early surgery are related to the operative field like less inflammation, less hematoma formation, and avoidance of the possibility of early callus formation which can further complicate surgery (11).

\section{Equipment preference card}

Recently, new material and technologies have been developed and enriched the field of surgical rib stabilization (8). Currently, the available hardware options for rib fixation can be categorized into five groups: (I) plating with bicortical screws, (II) absorbable plating, (III) Judet struts or Judet-like struts, (IV) Kirschner wires, and (V) intramedullary rods or splints (9). The most commonly used system is the 'plating with bicortical screws' technique, which has been frequently studied in scientific papers proving its favorable adaptability and ease of use $(9,11)$.

In our institution, we mainly use two different plating systems:

* The first system is RibFixBlu ${ }^{\mathrm{TM}}$ (Zimmer Biomet) and offers pre-contoured narrow $1.6 \mathrm{~mm}$ thick titanium plates that come with self-drilling $2.4 \mathrm{~mm}$ locking screws with a unique cancellous screw design. The available plates are straight 8 -hole as well as pre-contoured 12-, 16, and 24-hole plates. All the plates are made of pure titanium, which provides strength and flexibility; although it has to be taken into account that extensive bending may weaken them, which may result in a break in the future. The main advantages of this system are the self-drilling locking screws that can be implanted using a batterydriven pen-like automatic screw driver, which again safes quite some time compared to pre-drilling and positioning of the screws by hand with a mechanical screw driver. Furthermore, the plates and screws are cheaper compared to the MatrixRib ${ }^{\mathrm{TM}}$ described below.

* The second system, MatrixRib ${ }^{\mathrm{TM}}$ (DePuy Synthes, Johnson \& Johnson), provides both, universal (8 holes) and rib-specific plates with a $1.5 \mathrm{~mm}$ profile. The later are pre-contoured in a more sophisticated manner, not only respecting the different in-plane radiuses of different ribs, but also imitating the slight longitudinal twist of different ribs. These plates are made to fit either rib 3 ( 15 holes), ribs 4-5 (16 holes), ribs 6-7 (17 holes) or ribs 8-9 (18 holes), and are available for both sides [color coded to distinguish between plates for right-sided ribs (light red) and plates for the left side (light blue)]. This detailed pre-contouring works very good for most patients and safes quite some time since additional plate contouring during surgery is rarely required. Furthermore, since almost no additional manipulation of the plates is needed, they are supposedly more stable compared to a plate that has undergone excessive bending maneuvers in different planes. However, manufacturing of such plates is understandably more complicated and therefore the plates are significantly pricier than the ones of the RibFixBlu system. The locking screws have a diameter of $2.9 \mathrm{~mm}$ and require pre-drilling.

On the other hand, MatrixRib has some additional useful products to offer like for example intramedullary splints (available in widths of 3,4 or $5 \mathrm{~mm}$ ) as well as dedicated sternal plates.

The latter are markedly thicker plates that are available in different shapes ( $\mathrm{T}$ and I plates) as well as in the form of straight universal plates with 8,9 or 10 holes and a thickness of $2.8 \mathrm{~mm}$. These plates come in handy for fixation of sternal fractures in obese patients or in case of comminuted fractures, in which two parallel standard universal plates might not provide sufficient stability (Figures 2,3).

Besides one of the two abovementioned rib fixation systems, some other instruments are needed for the procedure as shown in Table 1. In case of suspected lung laceration (visible on CT-scan and/or air leak present with indwelling chest tube) and or hemopneumothorax, intraoperative thoracoscopy might be a worthwhile procedure while the patient is under general anesthesia.

Not only coagulated blood can be removed from the 

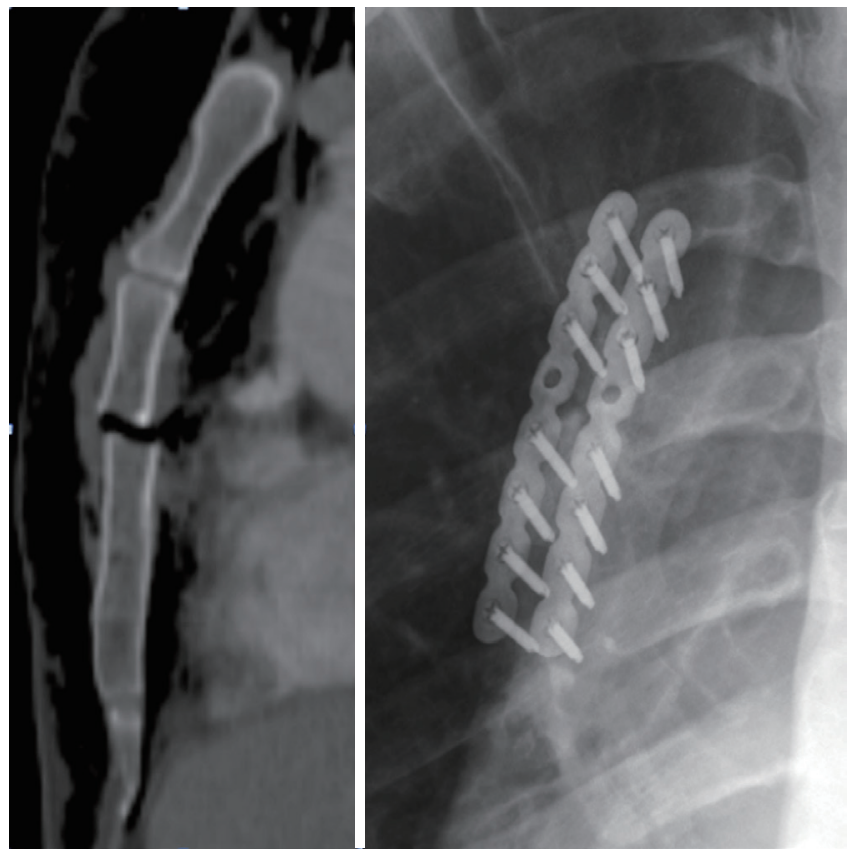

Figure 2 Surgical stabilization of simple sternal fracture in a slender patient with two parallel universal MatrixRib plates $(1.5 \mathrm{~mm}$ thick) (MatrixRib ${ }^{\mathrm{TM}}$ ).



Figure 3 Stabilization of comminuted sternal fracture after mechanical reanimation using two parallel $2.8 \mathrm{~mm}$ thick 8 -hole sternal plates (MatrixRib ${ }^{\mathrm{TM}}$ ).
Table 1 Equipment preference card for surgical rib fixation

\begin{tabular}{l} 
Retraction \\
Langenbeck retractors \\
Sharp retractors \\
Self-retaining retractors \\
Reposition of rib fracture \\
Bone reduction forceps \\
Raspatorium \\
Thoracoscopy \\
5 mm, 30 Camera \\
Lung grasper \\
Suction \\
Argon coagulator (Needle holder, open or thoracoscopic) \\
\hline
\end{tabular}

chest, but also small lung lacerations can be sealed with argon plasma coagulation or sutured with 4-0 PDS, if needed. As most lung lacerations are caused by fractured ribs piercing into the lung parenchyma, they can often be easily repaired through the same incision, which is used to expose the ribs (Figure 4).

\section{Procedure}

Usually surgical rib fixation requires general anesthesia. Furthermore, in cases of severe trauma with lung laceration and/or hemothorax, one-lung ventilation is helpful, which can be achieved by means of a double-lumen tube or a bronchial blocker. Single-lung ventilation facilitates evacuation of hemothorax as well as suturing of lung lacerations. However, in case of emergency surgery or other contraindications for single-lung ventilation, short phases of apnea may allow proper inspection of the chest cavity for hematoma evacuation and/or lung repair.

To access anteriorly located fractures, the patient is placed in a supine position. In the case of lateral or posterior fractures, the patient should be positioned in a lateral decubitus position. In some selected cases of posterior, paravertebral rib fractures, the patient needs to be put in a prone position. However, due to the thick layer of supporting soft tissue in the form of tendons and muscles in the paravertebral area, rib fractures in this region rarely require surgical repair. After the skin incision, subcutaneous flaps should be lifted in order to expose the muscles below. 

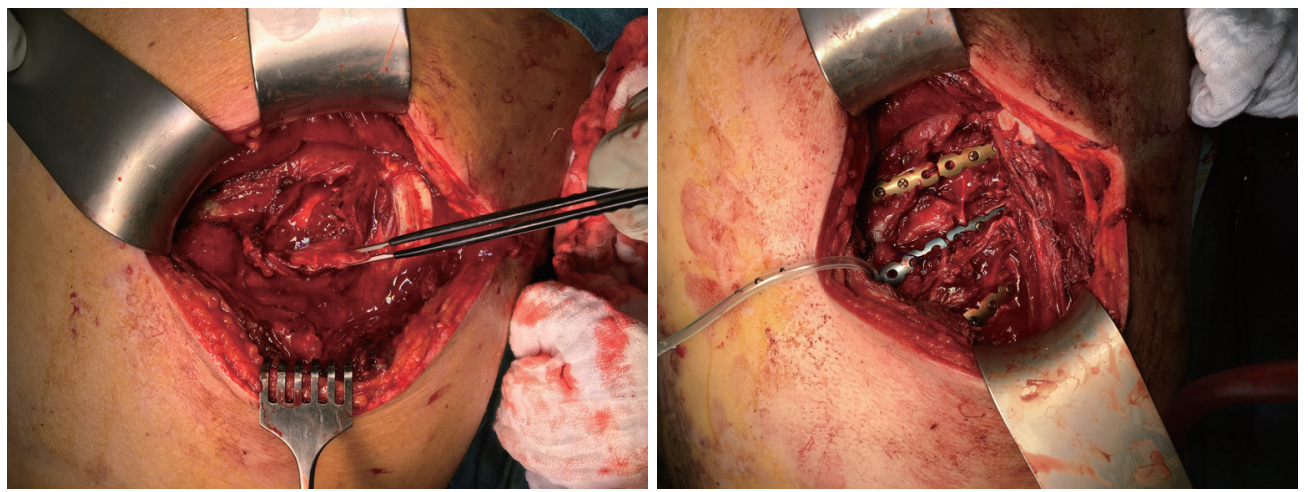

Figure 4 On the left: Lung laceration caused by piercing rib with visible air bubbles (just above tip of the forceps). On the right: After suturing of the lung and surgical rib fixation (using MatrixRib ${ }^{\mathrm{TM}}$ ).

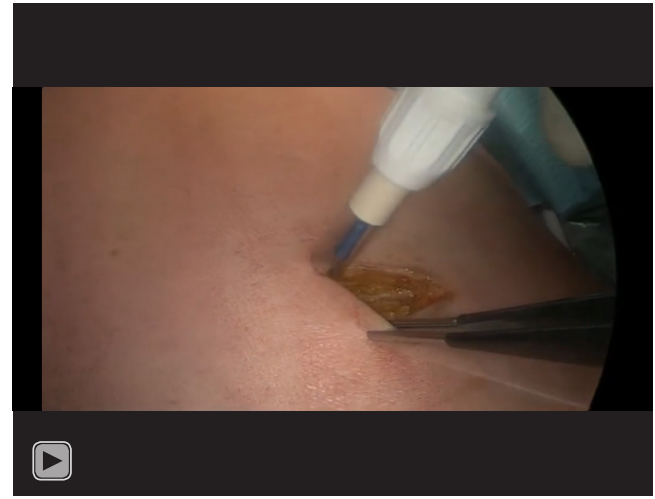

Video 1 Surgical technique of rib fixation (16).

Whenever possible, chest wall muscles should be split in the direction of their fibers in an attempt to spare them.

The surgeon must pay attention not to strip off the intercostal attachments or periosteum since this would not only cause additional unnecessary pain, but also impairs the ribs blood supply and with that, the subsequent healing of fractures.

Once the fracture is reduced and the rib contour is restored, the surgeon should take a precise measurement of the rib thickness using a caliper. If the screws are too long, they may damage the underlying lung, if too short the screws do not provide bicortical fixation, which may result in plate displacement.

According to the common fracture fixation principle, 3 screw holes on each side of the fracture should be occupied with bicortical screws. Depending of the type of fracture, the corresponding length of plate is chosenusually an 8-hole plate for simple fractures and longer plates for multifragmented fractures. The plate may need to be shortened to the desired length using a plate cutter and the resulting sharp edges at the site of cutting can be ground against the special rough groove in the plate cutter in order to make them smooth (as shown in the video). The plate then needs to be contoured according to the shape of the rib, either by simple manual bending-these low profile plates made of titanium are relatively easy to bend manually—or using dedicated plate bending instruments. Once the plate has its required shape it can either be hold on the rib with a forcep and fixated with a first screw, or held in position on the rib using a dedicated plate holding instrument. After fixation of the plate with one first screw, a slight rotation of the plate is still possible in order to perfectly align it with the rib. Then the remaining screws on one side of the fracture are placed. After that, the surgeon ensures that the reposition of the fracture is still preserved in an anatomical position before also inserting the screws on the other side of the fracture.

Before closure, hemostasis should be secured. If the pleural cavity was opened during surgery, a chest tube should be placed inside the thoracic cavity to drain any collection and/or air. The muscle layers are then approximated with 1-0 Vicryl, followed by a 3-0 or 4-0 Vicryl running suture of the subcutaneous tissue. Finally, the skin is closed using interrupted or intradermal absorbable running suture, depending on the surgeon's preference. We usually abstain from the use of additional subcutaneous and/ or submuscular suction drainages.

\section{Case example (Video 1)}

A 66-year-old male patient was referred two weeks after a bicycle accident with marked chest pain on the right and 


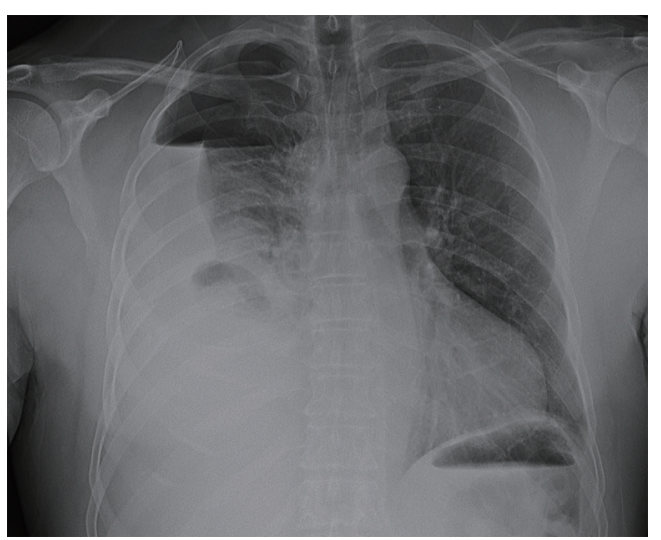

Figure 5 Chest X-ray showing right-sided hemopneumothorax.

dyspnea. Oxygen saturation was $92 \%$ on room air, but hemoglobin levels were low at $81 \mathrm{~g} / \mathrm{L}$ (normal reference 135-168 g/L). Chest X-ray showed hemopneumothorax on the right (Figure 5) and CT-scan confirmed a hemopneumothorax with simple fractures of the ribs 7-11 (Figure 6). Under general anesthesia with a double-lumen tube, the patient was put in a left lateral decubitus position. First, intrathoracic hematoma evacuation and pleural débridement (in case of pleural empyema stage I-II) was performed using a uniportal VATS approach through a 2-3 cm access in the $6 \mathrm{~h}$ intercostal space (Video 1). After placement of a chest tube, the fracture site(s) were identified and a skin incision was made over the eight rib. The latissimus dorsi muscles fibers were separated and the 8th rib was reduced and stabilized with an 8-hole plate, as were the adjacent ribs 7 and 9. For fracture fixation we used the RibFixBlu ${ }^{\mathrm{TM}}$ system, using one straight 8 -hole plate and one pre-contoured 16-hole plate that was divided in two. Once the chest wall was stable, the latissimus dorsi muscle was re-adapted and the soft tissue was closed. The lung was reventilated and the chest drain connected to a suction system at $-20 \mathrm{cmH}_{2} \mathrm{O}$.

\section{Role of team members}

The surgical team typically consists of two surgeons, occasionally three. The assistant stands on the opposite side of the table, facing the surgeon and mainly provides exposure of the fracture site. The anesthetist has mainly a crucial role for induction of one-lung ventilation in case thoracoscopy is needed. Furthermore, if a rib fixation system is used that requires pre-drilling of the screw holes, short phases of apnea may prevent the underlying lung from
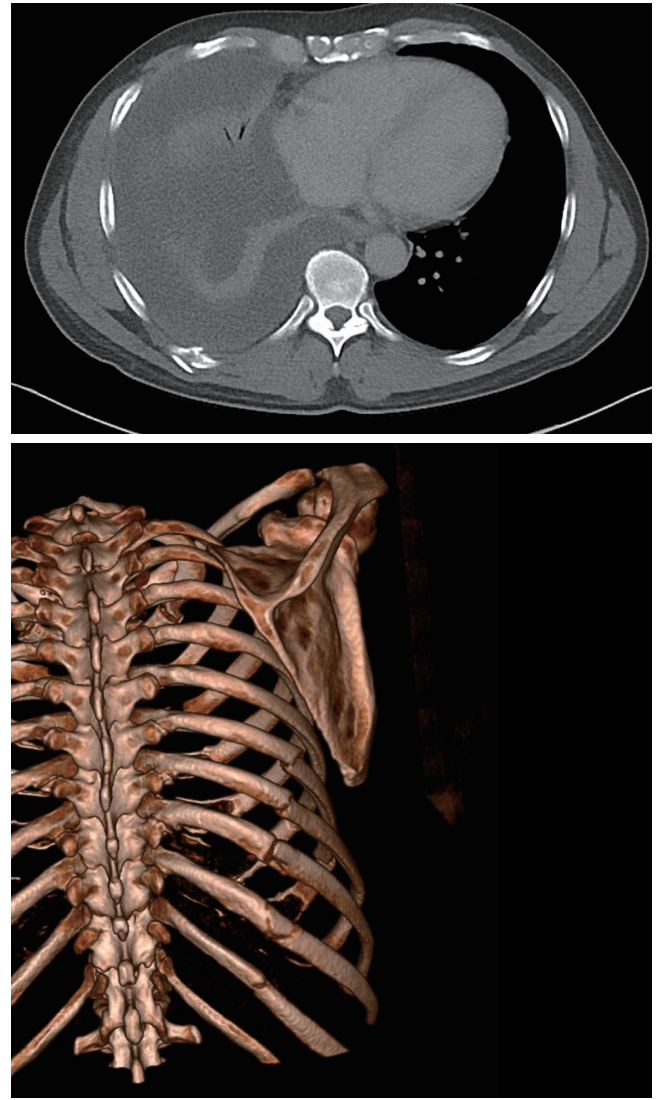

Figure 6 CT-scan showing hemothorax associated with posterolateral rib fractures of ribs 7-10 on the right [as shown on CT-3D reconstruction (right image)].

being 'scratched' by the tip of the drill.

\section{Post-operative management}

As especially in polytrauma patients rib fractures are not isolated injuries, the post-operative management should also focus on treating other accompanying injuries.

Studies show that rib fracture patients experience a decrease in the intensity of pain 72 hours postoperatively (5). During this period and after, the patient should be kept free of pain by regular analgesia. In addition, aggressive pulmonary hygiene should be applied to decrease the risk of subsequent pulmonary infections.

The usage of prophylactic anticoagulants should be balanced according to the mobility of the patient and the risk of bleeding related to other injuries.

The administration of antibiotics should be evaluated case by case. It is recommended to continue antibiotics in 
the postoperative period in case of an open fracture or other injuries that mandate their use.

\section{Tips, tricks and pitfalls}

* Preoperative planning:

Use a CT-scan for identification of the most relevant rib fractures, which need to be addressed during surgery. Accordingly the appropriate location of the incision and the required patient position can be determined. Furthermore, a decision should be made whether intraoperative thoracoscopy is deemed necessary [e.g., to evacuate clotted blood, to repair lung lacerations, diagnostic (diaphragmatic injury?)], since in this case single-lung ventilation should be available during surgery.

* Anesthesiologic considerations:

Apart from the abovementioned issue of one-lung ventilation, in patients with severe/extended injuries of the chest wall, and/or if even a thoracotomy for repair of other concomitant thoracic injuries might be needed, the preoperative insertion of an epidural catheter for peri- and postoperative analgesia should be considered.

* Surgical rib fixation:

- Not every rib that is fractured needs to be stabilized-it is often sufficient to fixate only every second rib or only the most displaced ones.

- Not every fracture in the same rib needs to be addressed-stability of the chest wall can usually be achieved once one of two fractures of one rib is fixed.

- Paravertebral rib fractures rarely need surgical fixation.

- Save time and money by dividing a pre-contoured 16-hole plate in two for fixation of two simple rib fractures.

* Postoperative care:

- Optimal pain control, daily respiratory exercises and early mobilization are key factors for a fast recovery.

\section{Conclusions}

Rib fractures are some of the most common traumatic injuries and are associated with significant morbidity and mortality. Despite the high number of cases worldwide, no internationally recognized guidelines regarding indications for surgical rib fixation are established yet. In this article, we share our experience in this field and we hope that it may help others in the decision making process and as a tool for the planning as well as the performance of such procedures. Furthermore, this article might be a start for setting up a consensus for surgical rib fracture fixation.

\section{Acknowledgments}

Funding: None.

\section{Footnote}

Provenance and Peer Review: This article was commissioned by the editorial office, Fournal of Visualized Surgery for the series "Chest Wall Traumas". The article has undergone external peer review.

Conflicts of Interest: All authors have completed the ICMJE uniform disclosure form (available at https://jovs. amegroups.com/article/view/10.21037/jovs.2019.11.04/ coif). The series "Chest Wall Traumas" was commissioned by the editorial office without any funding or sponsorship. FM served as the unpaid Guest Editor of the series. The authors have no other conflicts of interest to declare.

Ethical Statement: The authors are accountable for all aspects of the work in ensuring that questions related to the accuracy or integrity of any part of the work are appropriately investigated and resolved. All procedures performed in this study were in accordance with the Helsinki Declaration (as revised in 2013). The manuscript is waived from patient informed consent according to the ethics committee or institutional review board.

Open Access Statement: This is an Open Access article distributed in accordance with the Creative Commons Attribution-NonCommercial-NoDerivs 4.0 International License (CC BY-NC-ND 4.0), which permits the noncommercial replication and distribution of the article with the strict proviso that no changes or edits are made and the original work is properly cited (including links to both the formal publication through the relevant DOI and the license). See: https://creativecommons.org/licenses/by-nc-nd/4.0/.

\section{References}

1. de Campos JRM, White TW. Chest wall stabilization in trauma patients: Why, when, and how? J Thorac Dis 
2018;10:S951-62.

2. Baram A, Kakamad FH. Bilateral thoracic trauma; presentation and management, a case series. Ann Med Surg 2019;43:25-8.

3. Uchida K, Nishimura T, Takesada H, et al. Evaluation of efficacy and indications of surgical fixation for multiple rib fractures: a propensity-score matched analysis. Eur. J. Trauma Emerg Surg 2017;43:541-7.

4. Martin TJ, Eltorai AS, Dunn R, et al. Clinical management of rib fractures and methods for prevention of pulmonary complications: A review. Injury 2019;50:1159-65.

5. Liu X, Xiong K. Surgical management versus non-surgical management of rib fractures in chest trauma:a systematic review and meta-analysis. J Cardiothorac Surg 2019;14:45.

6. He Z, Zhang D, Xiao H, et al. The ideal methods for the management of rib fractures. J Thorac Dis 2019;11:S1078-89.

7. Beks RB, Peek J, de Jong MB, et al. Fixation of flail chest or multiple rib fractures: current evidence and how to proceed. A systematic review and meta-analysis. Eur J Trauma Emerg Surg 2019;45:631-44.

8. Zhang Q, Song L, Ning S, et al. Recent advances in rib fracture fixation. J Thorac Dis 2019;11:S1070-7.

doi: 10.21037 /jovs.2019.11.04

Cite this article as: Kocher GJ, Al-Hurani M, Minervini F. Surgical fixation of rib fractures: how I do it. J Vis Surg 2020;6:16.
9. De Moya M, Nirula R, Biffl W. Rib Fixation: Who, What, When? Trauma Surg Acute Care Open 2017;2:e000059.

10. Feng K, Yin D, Zheng W, et al. Treatment of Open Chest Rib Fractures with the Matrix Rib Internal Fixation System: A Case Report. Medicine (Baltimore) 2019;98:e15683.

11. Mitchell JD. Blunt chest trauma: Is there a place for rib stabilization? J Thorac Dis 2017;9:S211-7.

12. Senekjian L, Nirula R. Rib Fracture Fixation: Indications and Outcomes. Crit Care Clin 2017;33:153-65.

13. Kocher GJ, Sharafi S, Azenha LF, et al. Chest Wall Stabilization in Ventilator-Dependent Traumatic Flail Chest Patients: Who Benefits? Eur J Cardiothorac Surg 2017;51:696-701.

14. Kani KK, Mulcahy H, Porrino JA, et al. Thoracic Cage Injuries. Eur J Radiol 2018;110:225-32.

15. Lodhia JV, Konstantinidis K, Papagiannopoulos K. Surgical Management of Multiple Rib Fractures/Flail Chest. J Thorac Dis 2019;11:1668-75.

16. Kocher GJ, Al-Hurani M, Minervini F. Surgical technique of rib fixation. Asvide 2019;7:045. Available online: http:// www.asvide.com/watch/33085 